\title{
ARTICLE OPEN Creation of a library of induced pluripotent stem cells from Parkinsonian patients
}

\author{
Staffan Holmqvist ${ }^{1,2,3,9}$, Šárka Lehtonen ${ }^{4,9}$, Margarita Chumarina ${ }^{1,2,3}$, Katja A Puttonen ${ }^{4}$, Carla Azevedo ${ }^{1,2,3}$, Olga Lebedeva ${ }^{5}$, \\ Marika Ruponen ${ }^{4}$, Minna Oksanen ${ }^{4}$, Mehdi Djelloul ${ }^{1,2,3}$, Anna Collin ${ }^{6}$, Stefano Goldwurm ${ }^{7}$, Morten Meyer ${ }^{8}$, Maria Lagarkova $^{5}$, \\ Sergei Kiselev ${ }^{5}$, Jari Koistinaho ${ }^{4,10}$ and Laurent Roybon ${ }^{1,2,3,10}$
}

Induced pluripotent stem cells (iPSCs) are becoming an important source of pre-clinical models for research focusing on neurodegeneration. They offer the possibility for better understanding of common and divergent pathogenic mechanisms of brain diseases. Moreover, iPSCs provide a unique opportunity to develop personalized therapeutic strategies, as well as explore early pathogenic mechanisms, since they rely on the use of patients' own cells that are otherwise accessible only post-mortem, when neuronal death-related cellular pathways and processes are advanced and adaptive. Neurodegenerative diseases are in majority of unknown cause, but mutations in specific genes can lead to familial forms of these diseases. For example, mutations in the superoxide dismutase 1 gene lead to the motor neuron disease amyotrophic lateral sclerosis (ALS), while mutations in the SNCA gene encoding for alpha-synuclein protein lead to familial Parkinson's disease (PD). The generations of libraries of familial human ALS iPSC lines have been described, and the iPSCs rapidly became useful models for studying cell autonomous and non-cell autonomous mechanisms of the disease. Here we report the generation of a comprehensive library of iPSC lines of familial PD and an associated synucleinopathy, multiple system atrophy (MSA). In addition, we provide examples of relevant neural cell types these iPSC can be differentiated into, and which could be used to further explore early disease mechanisms. These human cellular models will be a valuable resource for identifying common and divergent mechanisms leading to neurodegeneration in PD and MSA.

npj Parkinson's Disease (2016) 2, 16009; doi:10.1038/npjparkd.2016.9; published online 2 June 2016

\section{INTRODUCTION}

The group of synucleinopathies consists mainly of three neurodegenerative diseases: dementia with Lewy bodies, Parkinson's disease (PD) and multiple system atrophy (MSA), the last being divided into Parkinsonian type MSA with degeneration of nigro-striatal dopamine neurons, and cerebellar type MSA with ataxic symptoms. The diseases share the same hallmark: intracellular aggregates composed in majority of a protein called alpha-synuclein (aSYN). The SNCA gene encodes for aSYN. When SNCA is mutated or multiplied, it leads to an early onset familial PD. ${ }^{1,2}$ Interestingly, while aSYN aggregates are found in neurons in dementia with Lewy bodies and PD, they are located in oligodendrocytes in MSA. ${ }^{3}$

Naturally, SNCA is expressed in neurons. A recent study from our laboratory showed that SNCA is also expressed at early stage of oligodendrocyte development. ${ }^{4}$ The role of aSYN in oligodendrocytes largely remains to be clarified. It is thought that under pathological condition, aSYN protein can aggregate in neurons and over time form Lewy bodies. ${ }^{5}$ Moreover, it was shown in experimental models that aSYN aggregates are toxic to neurons, ${ }^{6,7}$ and that a neuroblastoma cell line over-expressing human recombinant SNCA releases factors including aSYN, leading to glial reactivity, ${ }^{8}$ suggesting aSYN aggregates may cause neuronal injury. However, recent work evidenced that neuronal dysfunction and protein aggregation may be two independent events. ${ }^{9}$ Thus, although the progression of Lewy pathology throughout the brain may be due to a prion-like mechanism of cell-to-cell transfer of aSYN, ${ }^{10}$ it remains unclear what the initial molecular cascades leading to neuronal dysfunction are, and how they differ depending on the genetic background of the patients.

The reprogramming of human somatic cells using "stemness" transcription factors into induced pluripotent stem cells (iPSC) ${ }^{11}$ has revolutionized our way to approach scientific problems related to human diseases. Importantly, this discovery offers unlimited access to patient cells, which can subsequently be differentiated into relevant cell types to study early pathogenic mechanisms of neurodegeneration. ${ }^{12-17}$ Such iPSC-based research strategies could lead to the discovery of new therapeutic targets, biomarkers, and the development of humanized high-throughput models for drug discovery and environmental chemical safety assessment. ${ }^{12-17}$ Thus far, several studies utilizing iPSCbased models reported neuronal dysfunction reminiscent to mutations in PD-linked genes LRRK2 (leucine-rich repeat kinase 2), PINK (PTEN-induced putative kinase 1), and PARK2

\footnotetext{
${ }^{1}$ Stem Cell Laboratory for CNS Disease Modeling, Wallenberg Neuroscience Center, Department of Experimental Medical Science, BMC A10, Lund University, Lund, Sweden; ${ }^{2}$ Strategic Research Area MultiPark, Lund University, Lund, Sweden; ${ }^{3}$ Lund Stem Cell Center, Lund University, Lund, Sweden; ${ }^{4}$ Stem Cell Laboratory of Molecular Brain Research Group, Department of Neurobiology, A.I. Virtanen Institute, University of Eastern Finland, Kuopio, Finland; ${ }^{5}$ Russian Academy of Sciences, Vavilov Institute of General Genetics,

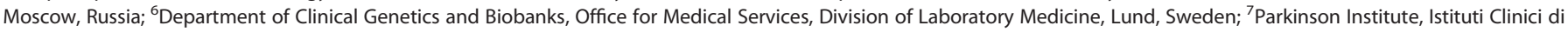
Perfezionamento, Milan, Italy and ${ }^{8}$ Department of Neurobiology Research, Institute of Molecular Medicine, University of Southern Denmark, Odense, Denmark.

Correspondence: J Koistinaho or L Roybon (jari.koistinaho@uef.fi or laurent.roybon@med.lu.se)

${ }^{9}$ These authors contributed equally to this work.

${ }^{10}$ These authors contributed equally to this work.

Received 23 December 2015; revised 1 April 2016; accepted 11 April 2016
} 
PD-iPSC library

S Holmqvist et al

\begin{tabular}{|c|c|c|c|c|c|c|c|c|}
\hline $\begin{array}{l}\text { Cell } \\
\text { type }\end{array}$ & $\begin{array}{l}\text { Tissue } \\
\text { type }\end{array}$ & Sex & $\begin{array}{l}\text { Cell } \\
\text { Line }\end{array}$ & $\begin{array}{l}\text { Molecular } \\
\text { analysis }\end{array}$ & Diagnosis & $\begin{array}{l}\text { Reprogramming } \\
\text { factors }\end{array}$ & Vector & Reference \\
\hline iPS & Fibroblast & $\mathrm{F}$ & UEF-1A & & Healthy subject & OSKM & Lentivirus & $15,26-28$ \\
\hline iPS & Fibroblast & $\mathrm{F}$ & UEF-2A & & Healthy subject & OSKM & Sendai virus & This study \\
\hline iPS & Fibroblast & $\mathrm{F}$ & UEF-2B & & Healthy subject & OSKM & Sendai virus & This study \\
\hline iPS & Fibroblast & $\mathrm{F}$ & UEF-2C & & Healthy subject & OSKM & Sendai virus & This study \\
\hline iPS & Fibroblast & M & UEF-3A & & Healthy subject & OSKM & Lentivirus & This study \\
\hline iPS & Fibroblast & M & UEF-3B & & Healthy subject & OSKM & Sendai virus & This study \\
\hline iPS & Fibroblast & M & UEF-4A & LRRK2 (p.G2019S) + GBA (p.N370S) & PD & OSKM & Sendai virus & This study \\
\hline iPS & Fibroblast & M & UEF-4B & LRRK2 (p.G2019S) + GBA (p.N370S) & PD & OSKM & Sendai virus & This study \\
\hline iPS & Fibroblast & M & UEF-5B & LRRK2 (p.G2019S) & PD & OSKM & Sendai virus & This study \\
\hline iPS & Fibroblast & M & UEF-5E & LRRK2 (p.G2019S) & PD & OSKM & Sendai virus & This study \\
\hline iPS & Fibroblast & M & UEF-5F & LRRK2 (p.G2019S) & PD & OSKM & Sendai virus & This study \\
\hline iPS & Fibroblast & M & UEF-5G & LRRK2 (p.G2019S) & PD & OSKM & Lentivirus & This study \\
\hline iPS & Fibroblast & $\mathrm{F}$ & CSC-1A & SNCA Duplication & PD & OSKM & Retrovirus & This study \\
\hline iPS & Fibroblast & $\mathrm{F}$ & CSC-1B & SNCA Duplication & PD & OSK & Retrovirus & This study \\
\hline iPS & Fibroblast & $\mathrm{F}$ & CSC-1C & SNCA Duplication & PD & OSK & Retrovirus & This study \\
\hline iPS & Fibroblast & $\mathrm{F}$ & CSC-1D & SNCA Duplication & PD & OSK & Retrovirus & This study \\
\hline iPS & Fibroblast & $\mathrm{F}$ & CSC-2A & SNCA Duplication & PD & OSK & Retrovirus & This study \\
\hline iPS & Fibroblast & $\mathrm{F}$ & $\mathrm{CSC}-2 \mathrm{~B}$ & SNCA Duplication & PD & OSKM & Retrovirus & This study \\
\hline iPS & Fibroblast & $\mathrm{F}$ & CSC $-2 C$ & SNCA Duplication & $\mathrm{PD}$ & OSKM & Retrovirus & This study \\
\hline iPS & Fibroblast & $\mathrm{F}$ & CSC-3A & SNCA Triplication & PD & OSK & Retrovirus & 4 \\
\hline iPS & Fibroblast & $\mathrm{F}$ & CSC-3B & SNCA Triplication & PD & OSK & Retrovirus & 4 \\
\hline iPS & Fibroblast & $\mathrm{F}$ & CSC-3G & SNCA Triplication & PD & OSK & Retrovirus & 4 \\
\hline iPS & Fibroblast & $\mathrm{F}$ & $\mathrm{CSC}-3 \mathrm{~S}$ & SNCA Triplication & PD & OSK & Retrovirus & 4 \\
\hline iPS & Fibroblast & $\mathrm{F}$ & CSC- $4 \mathrm{~A}$ & & MSA-C & OSK & Retrovirus & 4 \\
\hline iPS & Fibroblast & $\mathrm{F}$ & CSC-4B & & MSA-C & OSK & Retrovirus & 4 \\
\hline iPS & Fibroblast & $\mathrm{F}$ & CSC-6A & & MSA-P & OSKM & Retrovirus & 4 \\
\hline iPS & Fibroblast & $\mathrm{F}$ & CSC-7A & PARKIN (p.C273Y) & PD & OSK & Retrovirus & This study \\
\hline iPS & Fibroblast & $\mathrm{F}$ & CSC-7B & PARKIN (p.C273Y) & PD & OSK & Retrovirus & This study \\
\hline iPS & Fibroblast & $\mathrm{F}$ & CSC-8A & & Healthy subject & OSKM & Retrovirus & This study \\
\hline iPS & Fibroblast & $\mathrm{F}$ & CSC-8B & & Healthy subject & OSKM & Retrovirus & This study \\
\hline iPS & Fibroblast & $\mathrm{F}$ & CSC-8C & & Healthy subject & OSKM & Retrovirus & This study \\
\hline iPS & Fibroblast & $\mathrm{F}$ & $\mathrm{CSC}-8 \mathrm{~F}$ & & Healthy subject & OSKM & Retrovirus & This study \\
\hline iPS & Fibroblast & $\mathrm{F}$ & CSC-8S & & Healthy subject & OSKM & Retrovirus & This study \\
\hline iPS & Fibroblast & M & CSC-9A & & Healthy subject & OSKM & Retrovirus & 4 \\
\hline iPS & Fibroblast & M & CSC-9B & & Healthy subject & OSKM & Retrovirus & 4 \\
\hline iPS & Fibroblast & $\mathrm{F}$ & CSC-10A & GBA (p.L444P) & PD & OSKM & Sendai virus & This study \\
\hline iPS & Fibroblast & $\mathrm{F}$ & CSC-10B & GBA (p.L444P) & PD & OSKM & Sendai virus & This study \\
\hline iPS & Fibroblast & $\mathrm{F}$ & CSC-10C & GBA (p.L444P) & PD & OSKM & Sendai virus & This study \\
\hline iPS & Fibroblast & $\mathrm{F}$ & CSC-11A & PINK (p.Q456X) & PD & OSKM & Sendai virus & This study \\
\hline iPS & Fibroblast & $\mathrm{F}$ & CSC-11B & PINK (p.Q456X) & PD & OSKM & Sendai virus & This study \\
\hline iPS & Fibroblast & $\mathrm{F}$ & CSC-11C & PINK (p.Q456X) & PD & OSKM & Sendai virus & This study \\
\hline iPS & Fibroblast & M & CSC- $13 \mathrm{~A}$ & LRRK2 (p.G2019S) + GBA (p.N370S) & PD & OSKM & Sendai virus & This study \\
\hline iPS & Fibroblast & M & CSC-13C & LRRK2 (p.G2019S) + GBA (p.N370S) & PD & OSKM & Sendai virus & This study \\
\hline iPS & Fibroblast & M & CSC-14A & GBA (splicing mutation IVS $10+1 \mathrm{GT}$ ) & PD & OSKM & Sendai virus & This study \\
\hline iPS & Fibroblast & M & CSC-14B & GBA (splicing mutation IVS10+1GT) & PD & OSKM & Sendai virus & This study \\
\hline iPS & Fibroblast & M & CSC-14C & GBA (splicing mutation IVS $10+1 \mathrm{GT}$ ) & PD & OSKM & Sendai virus & This study \\
\hline iPS & Fibroblast & M & CSC-16B & PINK (p.Q456X) & PD & OSKM & Sendai virus & This study \\
\hline iPS & Fibroblast & M & CSC-16C & PINK (p.Q456X) & PD & OSKM & Sendai virus & This study \\
\hline iPS & Fibroblast & M & CSC-16D & PINK (p.Q456X) & PD & OSKM & Sendai virus & This study \\
\hline iPS & Fibroblast & M & CSC- $18 \mathrm{~A}$ & LRRK2 (p.R1441C) & PD & OSKM & Sendai virus & This study \\
\hline iPS & Fibroblast & M & CSC-18B & LRRK2 (p.R1441C) & PD & OSKM & Sendai virus & This study \\
\hline iPS & Fibroblast & M & CSC-18C & LRRK2 (p.R1441C) & PD & OSKM & Sendai virus & This study \\
\hline iPS & Fibroblast & M & CSC-19A & LRRK2 (p.G2019S) & PD & OSKM & Sendai virus & This study \\
\hline iPS & Fibroblast & M & CSC-19B & LRRK2 (p.G2019S) & PD & OSKM & Sendai virus & This study \\
\hline iPS & Fibroblast & M & CSC-19C & LRRK2 (p.G2019S) & PD & OSKM & Sendai virus & This study \\
\hline iPS & Fibroblast & M & CSC-20A & PARKIN (del Ex3) + GBA (p.N370S) & PD & OSKM & Sendai virus & This study \\
\hline iPS & Fibroblast & $\mathrm{M}$ & CSC-2OB & PARKIN (del Ex3) + GBA (p.N370S) & PD & OSKM & Sendai virus & This study \\
\hline iPS & Fibroblast & M & CSC-20C & PARKIN (del Ex3) + GBA (p.N370S) & PD & OSKM & Sendai virus & This study \\
\hline iPS & Fibroblast & $\mathrm{F}$ & CSC-21B & PARKIN (p.R275W) & PD & OSKM & Sendai virus & This study \\
\hline iPS & Fibroblast & $\mathrm{F}$ & CSC-21C & PARKIN (p.R275W) & PD & OSKM & Sendai virus & This study \\
\hline iPS & Fibroblast & $\mathrm{F}$ & CSC-22A & LRRK2 (p.G2019S) & PD & OSKM & Sendai virus & This study \\
\hline
\end{tabular}

Figure 1. Summary of human induced pluripotent stem cells generated. A total of 61 induced pluripotent stem cells (iPSC) lines are described in this study. These were reprogrammed by retroviral, lentiviral, or Sendai virus transduction of 'Yamanaka' factors. All iPSC lines were generated from human dermal fibroblasts. Somatic cells were sampled from individuals diagnosed with Parkinson's disease or multiple system atrophy, and healthy controls. The iPSC lines UEF-1A, CSC-3A, B, G and S, -4A and B, -6A and -9A, and B were previously characterized in refs 4, $15,26-28$.

(encodes PARKIN), ${ }^{18-21}$ or in the acid beta-glucocerebrosidase gene (GBA1), which encodes a lysosomal enzyme that is deficient in Gaucher's disease, and which renders a risk of developing PD. ${ }^{22,23}$ Therefore, iPSCs appear to be robust models for understanding early pathogenic events occurring in familial forms of PD. Importantly, iPSCs may provide an ideal platform for studying diseases where no genetic cause has thus far been identified, such as MSA and idiopathic PD. ${ }^{12-17}$
The generations of libraries of familial human ALS iPSC lines have been described; ${ }^{24,25}$ to date, none have been described for PD. Here, we report the generation of iPSCs carrying various mutations in PD-associated genes, as well as iPSCs generated from patients diagnosed with MSA and healthy controls (Figure 1). The lines have been generated and characterized in two laboratories located in the Nordic countries Finland and Sweden, allowing regrouping research efforts under the umbrella of several 
interconnected research centers, which focus their research on neurodegeneration. We present an exhaustive list of iPSC lines, and describe the tests we employed to validate their pluripotency and final selection. Moreover, we provide examples of relevant neural cell types, e.g., midbrain neural floor-plate progenitors, dopamine neurons, astrocytes, and oligodendrocytes that the iPSC lines can be differentiated into. Altogether, these human cellular models provide a unique resource to study PD and MSA.

\section{RESULTS}

IPSC lines generated in the Stem Cell Laboratory of Molecular Brain Research Group, at the University of Eastern Finland, in Finland

We present the characterization of iPSC lines generated by two reprogramming methods using either lentivirus or Sendai virus. We used the following nomenclature in description of University of Eastern Finland (UEF) lines: number = patient and letter = clone; e.g., UEF-2A $\rightarrow$ line generated at the UEF lab, patient 2, clone A. The skin biopsies were collected from individuals with confirmed diagnosis and mutation, and the fibroblast populations were transduced with viruses carrying genes encoding mouse Oct4, KIf4, SOX2, and CMyc (for UEF-1A line) or human OCT3/4, KLF4, SOX-2, and c-MYC (for UEF-3A and UEF-5G lines). The lines UEF-1A (i.e., UEFhfiPS1.4 in our previous reports ${ }^{15,26-28}$ ), UEF-3A and UEF-5G were generated with a polycistronic lentivirus carrying all the reprogramming factors in the same viral vector (STEMCCA) while the lines UEF-2A, $B$ and $C,-3 B,-4 A$ and $B$, and $-5 B, E, F$ and $G$, were transduced with four separate Sendai viruses, encoding the same pluripotency genes (Figure 1). Following the transduction with the four factors, we observed early morphological changes indicative of reprogramming (Figure 2a). An average of 3-6 embryonic stem cell (ESC)-like colonies were manually picked and expanded clonally. These clonal lines were cultured until about passage 10 prior to testing their pluripotency to ensure full maturation of hiPSCs. ${ }^{29,30}$ At that time point, we detected by using quantitative real-time $P C R$ pluripotency-promoting endogenous gene expression, including OCT3/4, SOX2, NANOG, KLF4, CMYC, and LIN28 in all our iPSC lines (Supplementary Figure S1), and confirmed the absence of the virally delivered transgenes (Figure $2 \mathrm{~b}$ and Supplementary Figure S1). All iPSC lines expressed several human ESC-associated antigens NANOG, SSEA4, TRA1-81, and OCT4 (Figure 2c), and the alkaline phosphatase staining was positive (Figure 2c). Chromosomal analysis from all iPS cell lines showed the normal karyotypes $46, X X$ or $46, X Y$ (Figures $2 d$ and 3 ) except of UEF-2A line where translocation in chromosome 2 and 9 was detected (Figure 3). In addition, the iPSC lines showed high telomerase activity when compared with their parent fibroblasts (Figure 2e).
All lines formed embryoid bodies when plated in suspension dishes (Figure 2f). Immunocytochemical analyses of embryoid bodies were performed after 10-14 days of culture and showed that each line had spontaneously differentiated into cell types representative of the three embryonic germ layers, including alpha-fetoprotein (AFP)-positive cells (endoderm), beta III-tubulin (B-III-TUB)-positive cells (ectoderm), and smooth muscle antibody (SMA)-positive cells (mesoderm; Figure $2 \mathrm{~g}$ ).

We adapted previously published protocols $\mathrm{s}^{31,32}$ to generate iPSC-derived mesencephalic dopaminergic neurons. The differentiation of iPSCs was started on human feeder layer. On day 14, small pieces of colonies were plated in an adherent culture. On day 20, the neuroepithelium differentiated into cells resembling neural cells. The neuroprogenitors were positive for LMX1A and FOXA2, confirming they acquired a midbrain floor-plate identity (Figure 2h). After 2 weeks of maturation, $\sim 40 \%$ of all cells were strongly immunoreactive for tyrosine hydroxylase (Figure 2i), and resembled tyrosine hydroxylase-positive neurons found in cultures derived from 5-week-old human embryonic ventral mesencephalic tissue (Figure 2h). Differentiation of astroglial progenitors and astrocytes from the iPSCs lines was adopted from a previously published protocol. ${ }^{33}$ Using this method we were able to generate a homogenous population of astrocytes within 4 months (Figure $2 \mathrm{~h}$ ).

IPSC lines generated in the Cell and Stem Cell Laboratory for CNS Disease Modeling, at the University of Lund, in Sweden

We present the characterization of iPSC lines generated using two distinct methods of reprogramming. All iPSC lines were generated from skin fibroblasts of individuals with confirmed diagnosis, obtained from open access resources. We adapted a protocol similar to that recently described, ${ }^{34}$ allowing us to reprogram up to 12 patient fibroblast samples at once, minimizing associated costs. The nomenclature used is similar to that implemented by UEF: number $=$ patient and letter $=$ clone; e.g., $\quad$ CSC-2A $\rightarrow$ line generated at the Cell and Stem Cell (CSC) lab, patient 2, clone $A$. The lines CSC- $1,-2,-3,-4,-6,-7,8-$, and -9 were generated by retroviral transduction of $3(\mathrm{OCT} 3 / 4, \mathrm{SOX} 2, \mathrm{KLF} 4)$ or 4 (the 3 plus c-MYC) factors; while the lines CSC-10, $-11,-13,-14,-16,-18,-19$, $-20,-21$, and -22 were generated by Sendai virus transduction of the four factors (Figure 1). Putative iPSC colonies were picked only if presenting a well-defined pluripotent stem cell-like morphology (Figure 4a). Unless fibroblasts displayed low reprogramming efficiency, an average of 24 colonies was picked per patient reprogrammed fibroblasts. These were subsequently expanded onto irradiated mouse embryonic fibroblasts (using a 24-well plate format) for up to 4 passages, prior to being cryopreserved.

We commonly thaw 3 putative iPSC clones for each patient lines generated, grow all clonal cell lines in 6-well plates for up to 10

Figure 2. Tests of pluripotency applied to the induced pluripotent stem cells (iPSC) lines generated in the Stem Cell Laboratory of Molecular Brain Research Group, at the University of Eastern Finland (UEF), in Finland. (a) Representative bright field images of human fibroblasts prior to transduction and after the transduction. The putative iPSC colony shows a hES cell-like morphology. (b) The expression levels of Sendai virus in iPSC lines UEF-2B and C (at passage 17; 15), UEF-3B (at passage 15), UEF-4A and B (at passage 17; 15), and UEF-5B, E and F (at passage 18; 15; 16). The levels are compared to non-transduced fibroblast and to hES cells. (c) Representative fluorescence images of iPSC lines stained for the pluripotent markers OCT4, NANOG, TRA1-80 and SSEA4. IPSC colonies stain positive for alkaline phosphatase activity. Images are shown for iPSC line UEF-5F. Scale bars represent $100 \mu \mathrm{m}$. (d) Karyogram of iPSC line UEF-2B shows pairs of chromosomes stained using Giemsa (Gbanding). (f) IPSC lines form embryoid bodies (EBs) grown in low-adherent plates for 2 weeks (representative image shown for iPSC line UEF-4A, 5B,F). (g) Differentiated EBs generate cells of the three germ layers, immunopositive for alpha-fetoprotein (AFP) (endoderm), smooth muscle antibody (SMA) (mesoderm), and beta III-tubulin (B-III-TUB) (ectoderm); nuclei are counterstained with 4,6-diamidino-2-2phenylindole (DAPI; shown for iPS lines UEF-3B and UEF-4A). (e) Detection of telomerase activity using TRAPeze telomerase detection kit. (h) Representative images of iPSC lines differentiated towards neural, neuronal, and glial fates. IPSC lines differentiated for 20 days are positive for LMX1A and FOXA2 (midbrain neural progenitors, UEF-1A); when progenitors are kept for two additional weeks in maturation medium, they differentiate into tyrosine hydroxylase (TH)-expressing neurons (shown for iPSC line UEF-4B) that co-label with FOXA2 (shown for iPSC line UEF-2C). Undifferentiated neural progenitors cultured for 4 additional months generate glial fibrillary acidic protein (GFAP)-expressing astrocytes (shown for iPSC line UEF-5B). TH-positive and GFAP-positive cells in a human-derived ventral mesencephalic (hVM) culture served as a reference. Nuclei are counterstained with DAPI. Scale bars represent 100, 50 and $20 \mu \mathrm{m}$. (i) Differentiated iPS cells towards tyrosine hydroxylase positive $(\mathrm{TH}+$ ) cells at day 35 . Data are expressed as mean $\%$ of $\mathrm{TH}+$ cells compared to DAPI $\pm \mathrm{SD}$. 
(some times more) passages (with one simultaneous passage per week), and perform within the latest passages, in addition to finger print analysis, a battery of tests allowing us to determine the efficiency of reprogramming from putative to well-established/mature iPSC lines. The tests comprise: (1) expression of nuclear and cell surface markers reminiscent to pluripotent stem cell stage (OCT4, NANOG, TRA1-80 and SSEA4), (2) alkaline phosphatase activity, (3) telomerase activity, (4) embryoid body formation, (5) loss of viral agents or downregulation of viral transgenes, and (6) differentiation towards the three germ layers. The lines can further be tested for their ability to generate midbrain neural cells types: LMX1A/FOXA2 co-expressing neural floor-plate progenitors, FOXA2/tyrosine hydroxylase-expressing neurons, glial fibrillary acidic protein-expressing astrocytes, or O4-positive oligodendrocytes, which can be used for studies of development and disease phenotypes. All these tests are

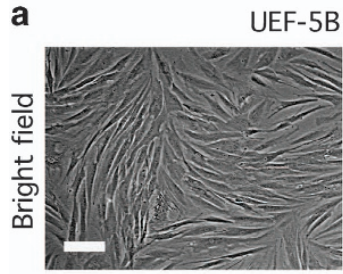

Fibroblasts

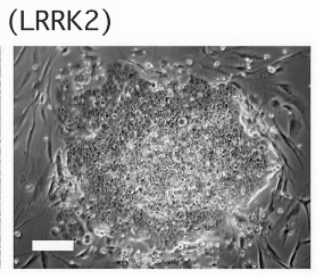

Colony at picking

b

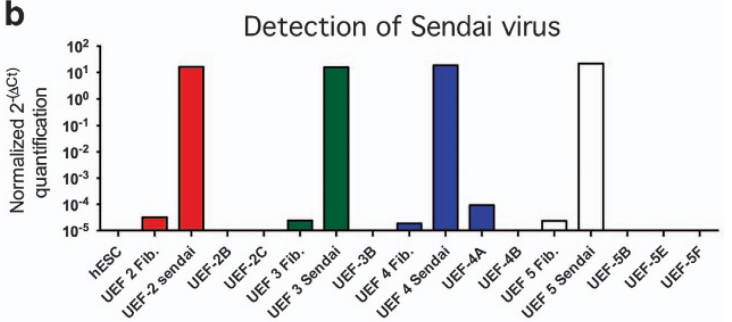

C

OCT4

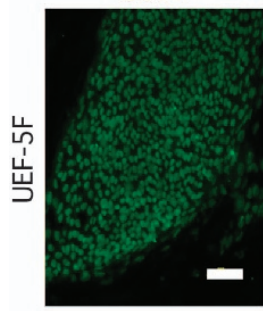

NANOG

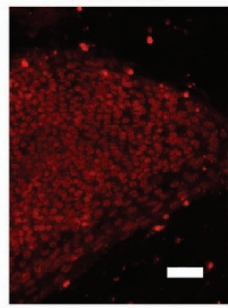

TRA $1-80$
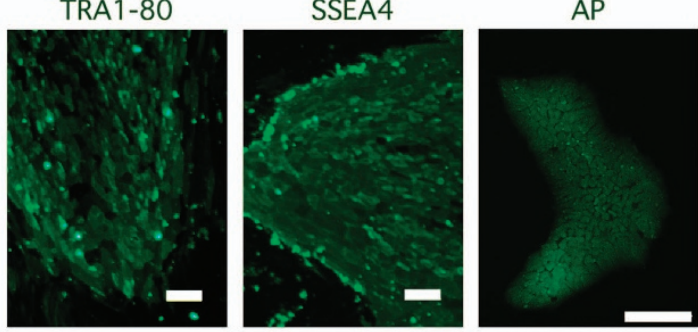

Bright field

d

UEF-2B

影

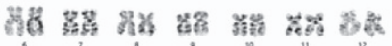

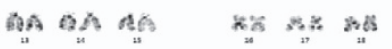

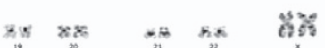

e

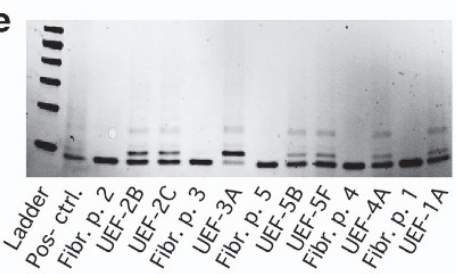

f
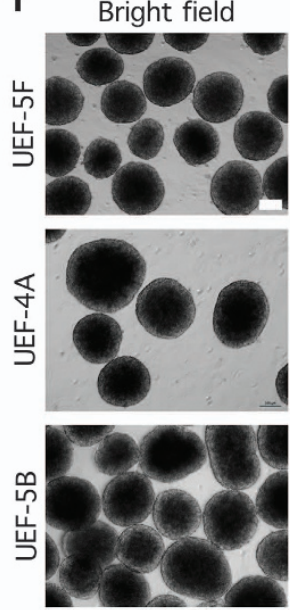

\section{g}
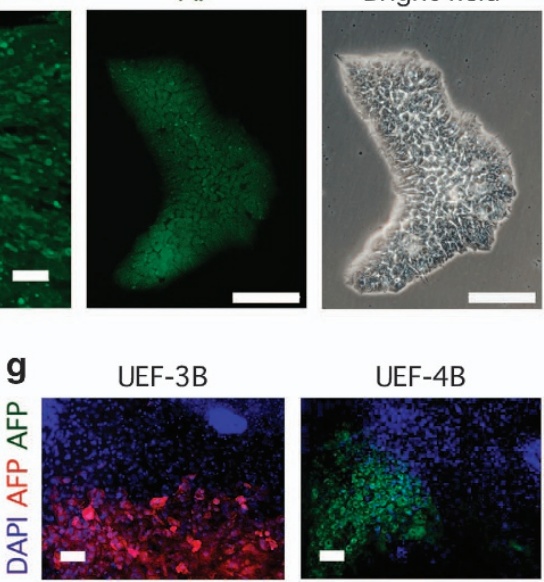

h
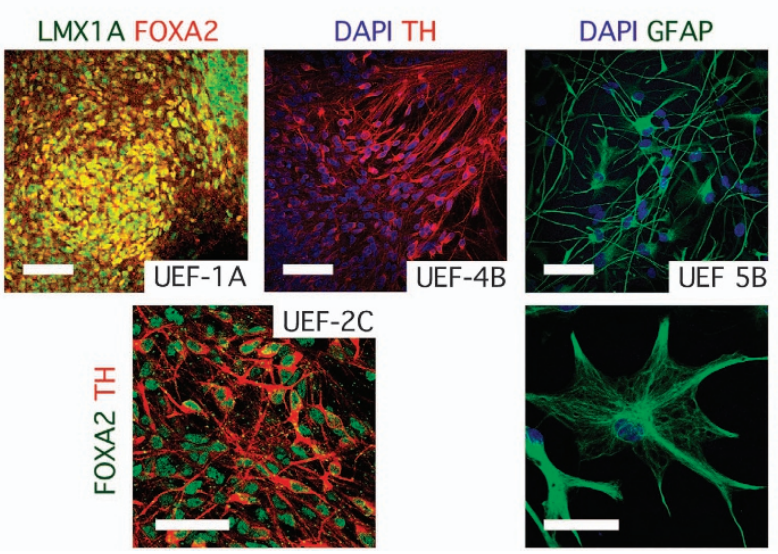
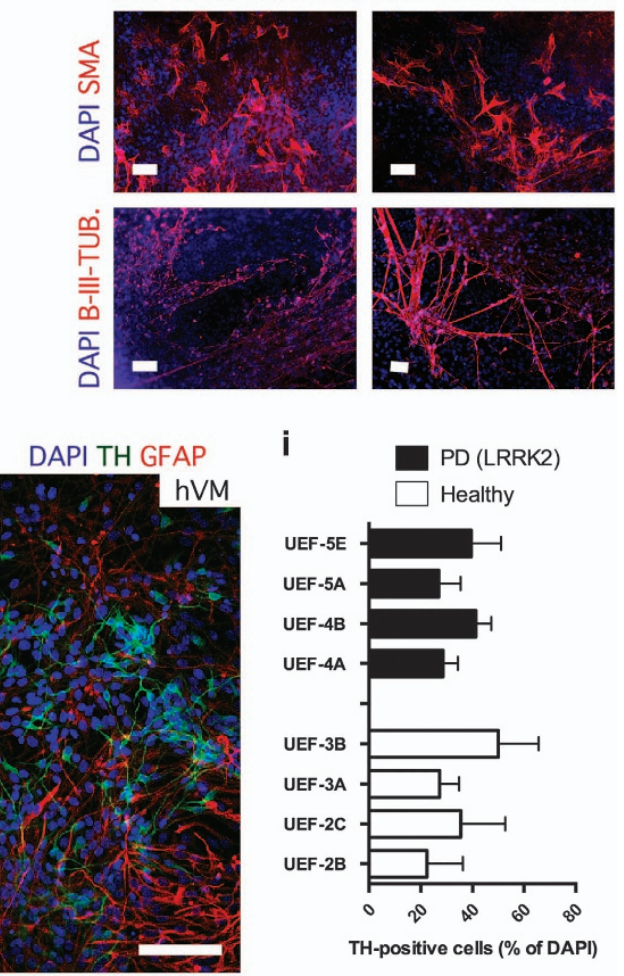

Figure 2. For caption please refer page 3. 


\begin{tabular}{|c|c|c|c|}
\hline Cell line & Vector & Passage & Genetic analysis \\
\hline UEF-1A & Lentivirus & P10 & $46, X X[20]$ \\
\hline UEF-2A & Sendai virus & P15 & $46, X X, t(2 ; 9)(q 33 ; q 34)[6]$ \\
\hline UEF-2A & Sendai virus & P35 & $46, X X, t(2 ; 9)(q 33 ; q 34)[7]$ \\
\hline UEF-2B & Sendai virus & P14 & $46, X X[20]$ \\
\hline UEF-2C & Sendai virus & P21 & $46, X \times[20]$ \\
\hline UEF-3A & Lentivirus & P12 & $46, X Y[20]$ \\
\hline UEF-3B & Sendai virus & P10 & $46, X Y[20]$ \\
\hline UEF-4A & Sendai virus & P8 & $46, X Y[20]$ \\
\hline UEF-4B & Sendai virus & P15 & $46, \mathrm{XY}[5]$ \\
\hline UEF-5B & Sendai virus & P20 & $46, X Y[5]$ \\
\hline UEF-5E & Sendai virus & P9 & $46, \mathrm{XY}[20]$ \\
\hline UEF-5F & Sendai virus & P25 & $46, X Y[5]$ \\
\hline UEF-5G & Lentivirus & P15 & $46, \mathrm{XY}[20]$ \\
\hline CSC-1A & Retrovirus & P20 & $46, X X[17]$ \\
\hline CSC-1B & Retrovirus & P20 & $47,+2-3 r$, inc $[2] / 46, x x[18]$ \\
\hline CSC-1C & Retrovirus & P17 & $47, X X,+18[3] / 46, X X[17]$ \\
\hline CSC-1D & Retrovirus & P15 & $46, X X[15]$ \\
\hline CSC-2A & Retrovirus & P20 & $46, X X, \operatorname{add}(18)(\mathrm{p} 11),-22,+\operatorname{mar}[23]$ \\
\hline CSC-2B & Retrovirus & P17 & $46, \mathrm{XX},-4,-22[\mathrm{cp} 4] / 46, \mathrm{XX}[21]$ \\
\hline CSC-2C & Retrovirus & P17 & $46, X X[24]$ \\
\hline CSC-3A & Retrovirus & P20 & $46, \mathrm{XX}, \mathrm{t}(11 ; 17)(\mathrm{p} 15 ; \mathrm{q} 25)[11]$ \\
\hline CSC-3B & Retrovirus & P27 & $46, \mathrm{XX}, \mathrm{t}(11 ; 17)(\mathrm{p} 14 ; \mathrm{q} 25)[23] / 46, \mathrm{XX}[2]$ \\
\hline CSC-3G & Retrovirus & P15 & $46, x X[25]$ \\
\hline CSC-3S & Retrovirus & P13 & $46, X X[25]$ \\
\hline CSC-4A & Retrovirus & P23 & $39-46, X X,-2,-11[\mathrm{cp} 3] / 46, X X[18]$ \\
\hline CSC-4B & Retrovirus & P13 & $46, X X[25]$ \\
\hline CSC-6A & Retrovirus & P15 & $46, X \times[24]$ \\
\hline CSC-7A & Retrovirus & P13 & $46, X X[18]$ \\
\hline CSC-7B & Retrovirus & P9 & $46, X X[25]$ \\
\hline CSC-8A & Retrovirus & P12 & $46, X X, \operatorname{inv}(6)(p 23 q 21)[25]$ \\
\hline CSC-8B & Retrovirus & P18 & $46, X X, \operatorname{inv}(6)(p 25 q 21)[25]$ \\
\hline CSC-8C & Retrovirus & P7 & $46, X X, \operatorname{inv}(6)(p 21 q 21)[15]$ \\
\hline CSC-8F & Retrovirus & P8 & $46, X X, \operatorname{inv}(6)(p 25 q 21)[14]$ \\
\hline CSC-8S & Retrovirus & P7 & $46, X X[9]$ \\
\hline CSC-9A & Retrovirus & P13 & $46, \operatorname{XY}[25]$ \\
\hline CSC-9B & Retrovirus & P20 & $45-47, \mathrm{XY},+\operatorname{mar}[\mathrm{cp} 5] / 46, \mathrm{XY}[20]$ \\
\hline CSC-10A & Sendai virus & P8 & $46, X \times[24]$ \\
\hline CSC-10B & Sendai virus & P7 & $46, X \times[24]$ \\
\hline CSC-10C & Sendai virus & P8 & $46, X \times[13]$ \\
\hline CSC-11A & Sendai virus & P11 & $46, X \times[12]$ \\
\hline CSC-11B & Sendai virus & P10 & $46, x \times[12]$ \\
\hline CSC-11C & Sendai virus & P10 & $46, \mathrm{XX}, \mathrm{t}(2 ; 16)(\mathrm{q} 21 ; \mathrm{p} 12)[15]$ \\
\hline CSC-13A & Sendai virus & P7 & $46, X Y, \operatorname{add}(7)(q ? 22)[15]$ \\
\hline CSC $-13 \mathrm{C}$ & Sendai virus & P7 & $46, X Y[15]$ \\
\hline CSC-14A & Sendai virus & P10 & $46, \mathrm{XY}, \mathrm{t}(2 ; 12 ; 18)(\mathrm{q} 23 ; \mathrm{q} 15 ; \mathrm{q} 21)[16]$ \\
\hline CSC-14B & Sendai virus & P10 & $46, \mathrm{XY}, \mathrm{t}(2 ; 12 ; 18)(\mathrm{q} 23 ; \mathrm{q} 15 ; \mathrm{q} 21)[15]$ \\
\hline CSC-14C & Sendai virus & P11 & $46, X Y, t(2 ; 12 ; 18)(q 23 ; q 15 ; q 21)[15]$ \\
\hline CSC-16B & Sendai virus & P8 & $46, \mathrm{XY}[15]$ \\
\hline CSC-16C & Sendai virus & P7 & $46, X Y[15]$ \\
\hline CSC-16D & Sendai virus & P7 & $46, X Y[15]$ \\
\hline CSC-18A & Sendai virus & P12 & $46, X Y[25]$ \\
\hline CSC-18B & Sendai virus & P12 & $46, X Y[10]$ \\
\hline CSC-18C & Sendai virus & P12 & $46, X Y[15]$ \\
\hline CSC-19A & Sendai virus & P12 & $46, X Y[16]$ \\
\hline CSC-19B & Sendai virus & P7 & $46, X Y[16]$ \\
\hline CSC-19C & Sendai virus & P17 & $46, X Y[15]$ \\
\hline CSC-20A & Sendai virus & P9 & $46, X Y[15]$ \\
\hline CSC-20B & Sendai virus & P9 & $46, X Y[15]$ \\
\hline CSC-20C & Sendai virus & P9 & $46, X Y[15]$ \\
\hline CSC-21B & Sendai virus & P7 & $46, X X[14]$ \\
\hline CSC-21C & Sendai virus & P17 & $46, X X$, add $(19)(q 13)[15]$ \\
\hline & & & \\
\hline
\end{tabular}

b

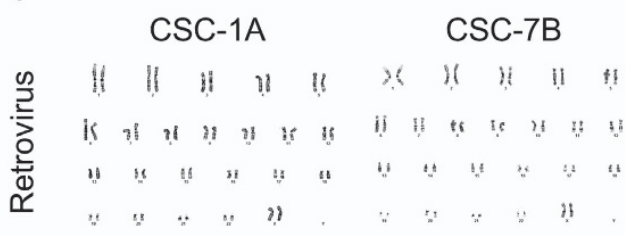

CSC-11A

CSC-10B

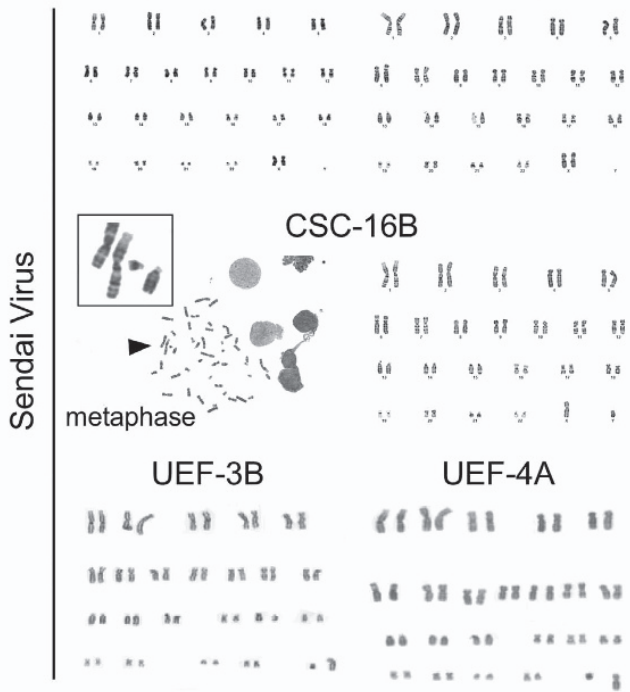

UEF-3A

UEF-5G

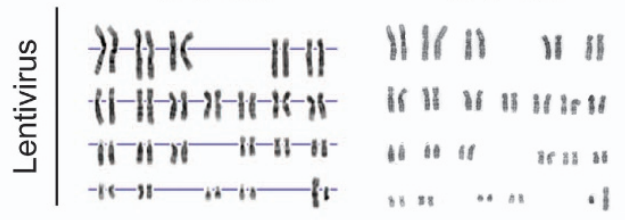

C

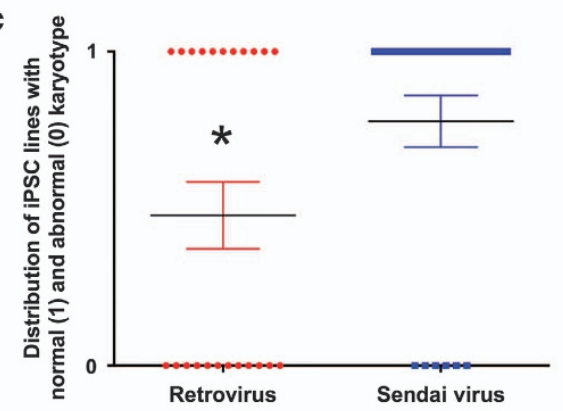

NOTE: parent fibroblasts of lines 14A, B and C are 46,XY,t(2;12;18)(q23;q15;q21)[3]/46,XY[10]

Figure 3. Cytogenic analysis of the induced pluripotent stem cells (iPSC) lines. (a) List of the iPSCs lines and corresponding karyotypes based on G-banding analysis. Note: parent fibroblasts used to generate the lines CSC-14A, CSC-14B and CSC-14C, are a mosaic of abnormal (46,XY,t $(2 ; 12 ; 18)(q 23 ; q 15 ; q 21)$ and normal $(46, X Y)$ cells. (b) Karyograms of iPSC lines reprogramed using retrovirus (CSC-1A and CSC-7B), Sendai virus (CSC-11A, CSC-10B, CSC-16B, UEF-3B and UEF-4A), and lentivirus (UEF-3A and UEF-5G) transduction. Representative image of metaphase (shown for CSC-16B) used for generating karyograms. (c) Higher numbers of iPSC lines with abnormal karyotyping are generated when parent fibroblasts are reprogrammed using retroviral transduction. Unpaired $t$-test revealed significance between the two groups compared $\left({ }^{*} P<0.05\right)$; lentivirus group was not included in the analysis because $n=3$ only. Note: analysis performed for Cell and Stem Cell (CSC) laboratory iPSC lines only; iPSC lines CSC-14A, -14B and -14C carrying the same chromosomal abnormality in all clones tested were excluded from the distribution analysis, as they reflected a constitutional chromosomal change present in the parent fibroblasts. 
performed in vitro. In addition, the lines are tested for their karyotype stability, and the tests are performed including a human ESC line (HuES3 and/or HuES13) as positive control. In some assays, e.g., determination of telomerase activity, both embryonic stem cells and parent fibroblasts can be used as positive or negative controls, as we previously reported, ${ }^{4}$ as well as heatinactivated samples.
Sendai virus transduction is advantageous in many ways, as it leads to high efficiency reprogramming when compared with retroviral delivery method of reprogramming, and it is nonintegrative. ${ }^{35}$ Interestingly, two clonal cell lines (CSC-11C at passage 13 and CSC-13A at passage 8) showed resistance to Sendai virus elimination over passages (Figure $4 b$ and Figure 5). Consequently, these failed reprogrammed clones only partially

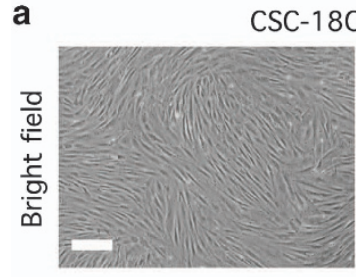

Fibroblasts
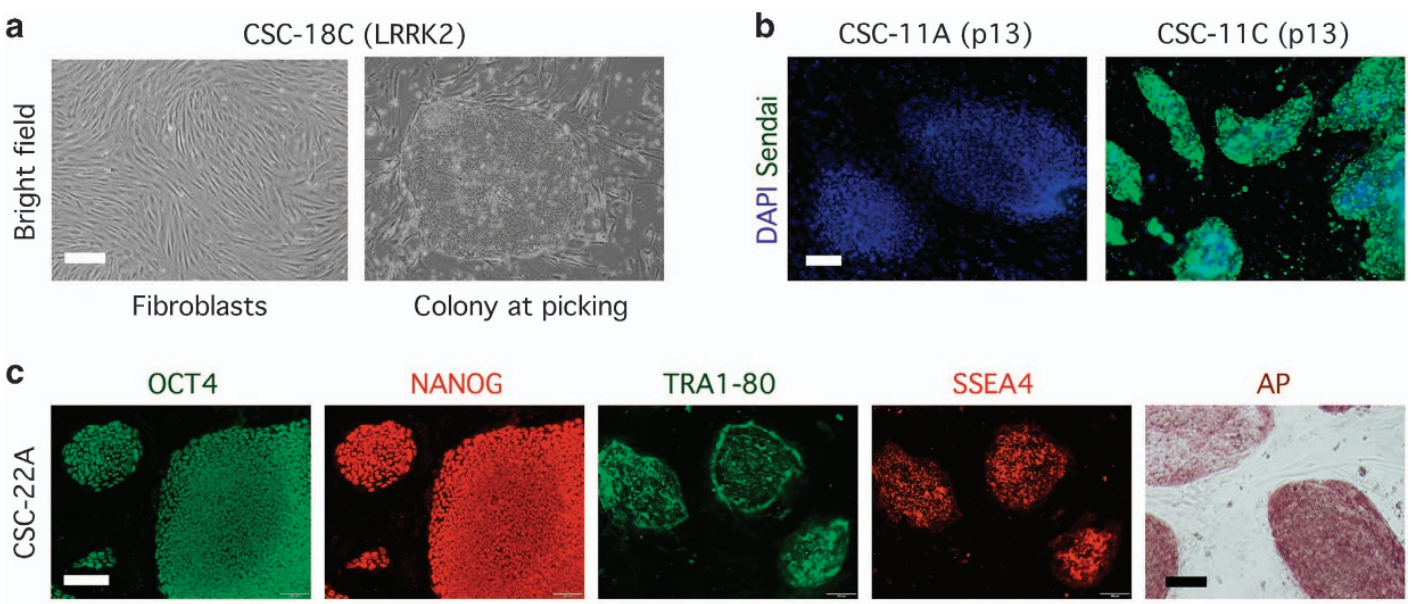

d

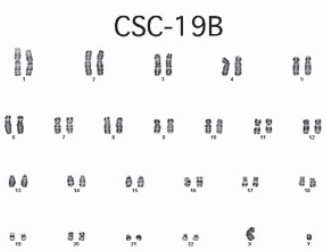

e
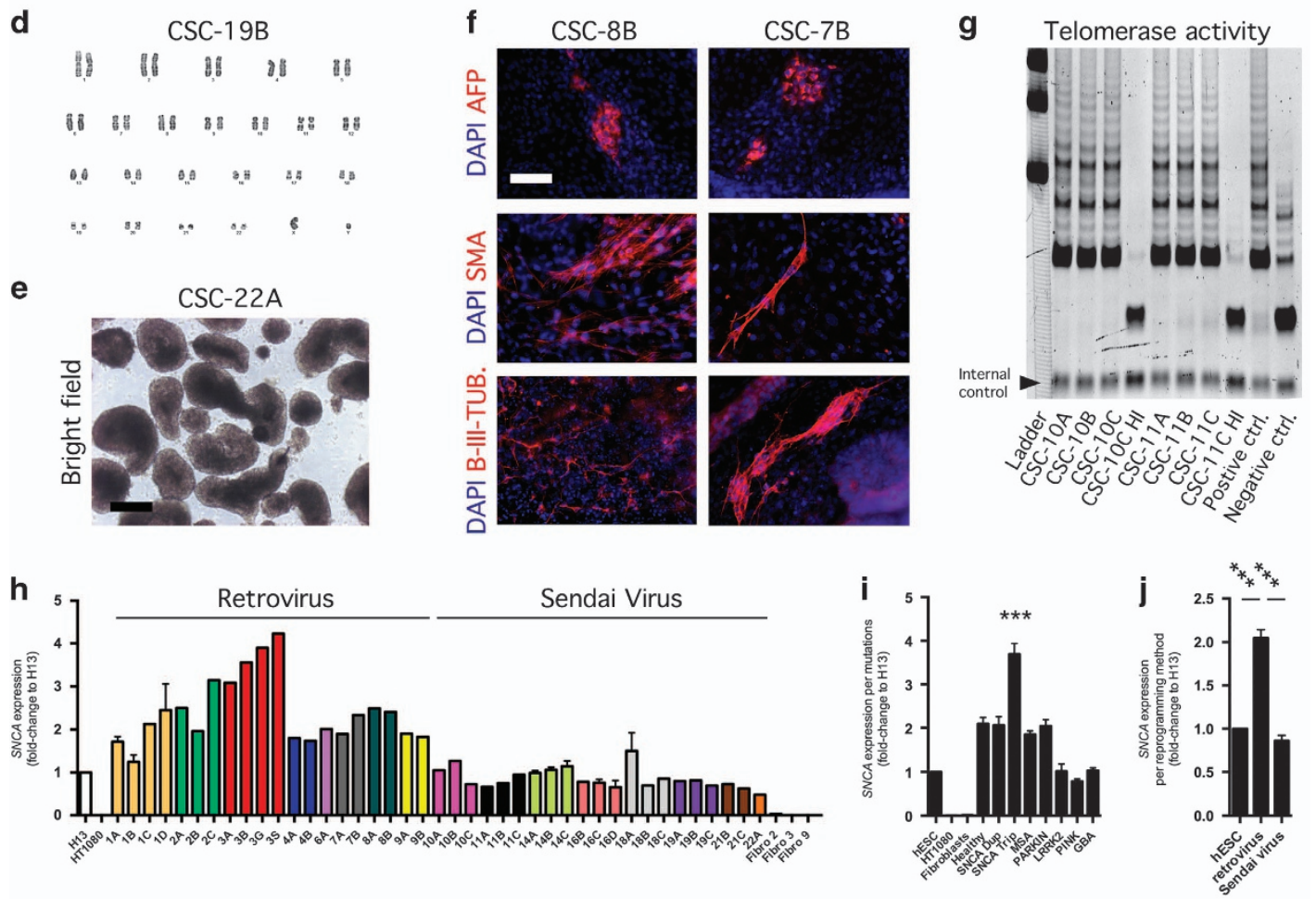

k
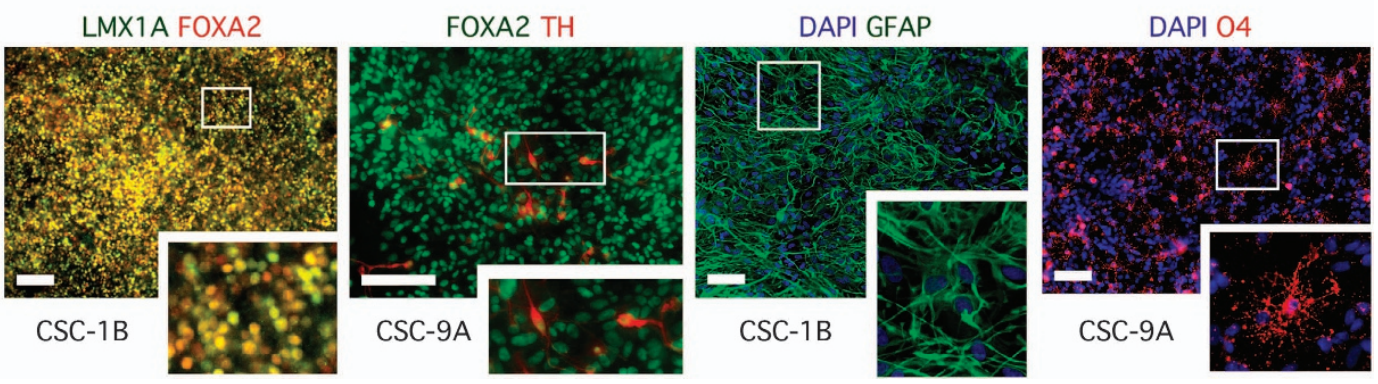
passed most of the tests (Figure 5). Importantly, all other lines, including those reprogrammed using retroviral transduction, showed immunoreactivity for the pluripotency markers OCT4, NANOG, TRA1-80, and SSEA4. Almost all lines had completely downregulated viral transgene expression, with the exception of line CSC-7B, which had a strong expression of viral OCT4 (Figure 4c, Supplementary Figure S2, and Figure 5); moreover, the lines displayed a robust alkaline phosphatase activity (Figure 4c), which we observed weaker for CSC-11C and CSC-13A. Although we did not perform rigorous side-by-side comparisons of the reprogramming methods, we observed that reprogramming by means of Sendai virus transduction compared to retrovirus transduction led to less karyotyping abnormalities (Figure $4 \mathrm{~d}$ and Figure 3), as recently described. ${ }^{36}$ Nevertheless, the clonal cell lines could generate embryoid bodies when cultured on non-adherent surfaces, in Wicell medium supplemented with basic fibroblast growth factor (Figure 4e), and subsequently differentiate into all three embryonic germ layers when exposed to fetal bovine serum, as detected by the presence of AFP-positive, SMA-positive, and B-III-TUB-positive cells, after 2 weeks in vitro (Figure 4f). Finally, we confirmed high telomerase activity for the iPSCs (Figure $4 \mathrm{~g}$ ), as well as higher expression of SNCA gene for the clones CSC-3A, -3B, -3G, and -3S (SNCA triplication), regardless whether they were generated using three or four factors and if they carried karyotype abnormalities (Figure $4 \mathrm{~h}$ and Figure 3). Interestingly, the iPSC lines reprogrammed by retroviral transduction displayed higher basal level of expression of SNCA gene compared with hESC control and iPSC lines generated by Sendai virus transduction; this observation required the exclusion from the analysis of the lines harboring duplication and triplication of SNCA gene (Figure $4 \mathrm{j}$ ), and may underlie a possible leakiness in expression of the integrated transgenes (Supplementary Figure S2).

We adapted our previous published protocols ${ }^{4,37}$ to generate iPSC-derived neural progenies. Thus, we could generate midbrain neural floor-plate LMX1A/FOXA2-co-expressing progenitors, which after subsequent differentiation gave rise to FOXA2/tyrosine hydroxylase-expressing neurons and glial fibrillary acidic proteinexpressing astrocytes (Figure 4k). IPSCs could also be differentiated into O4-positive oligodendrocytes (Figure 4k).

\section{DISCUSSION}

It was only less than a decade ago that successful reprogramming of somatic cells into ESC-like cells was reported. Two initial studies described that treating permeabilized human 293T cells with carcinoma nuclear cell extract, and overexpression of four pluripotent genes in dividing mouse fibroblasts, could reprogram somatic cells back to an ESC-like pluripotent stage. ${ }^{38,39}$ The first method was adapted from previous report of the same group, showing that human 293T cells expressed T-cell functions when reprogrammed using primary human T-cell extract. ${ }^{40}$ The second method, employing overexpression of rodent pluripotent genes Sox2, Oct4, KIf4, and c-Myc, allowed large-scale generation of the so-called iPSCs, and is now routinely used for the reprogramming of patient somatic cells for 'modeling diseases in the dish'.

Various techniques exist to generate iPSC; they are commonly subdivided into genome-integrating and genome non-integrating approaches. These technologies rely on the use of different vectors ${ }^{35}$ and they have been extensively studied and improved during the last years. ${ }^{41,42}$ Moreover, much effort to identify chemical compounds that could substitute the reprogramming factors and the use of vectors is ongoing. ${ }^{43}$ Interestingly, when we compared the expression of SNCA in iPSC lines generated using two different approaches, we found higher level of SNCA expression in iPSC lines generated using a genomeintegrating approach, when compared with iPSC lines generated by Sendai virus transduction; this analysis excluded the lines carrying multiplications of the gene of interest. Thus, it will be important in subsequent studies to verify that variability is not added by the reprogramming method employed to generate the iPSCs, to that already existing amongst the different clonal cell lines generated from a single patient biopsy sample. This may prevent the identification of subtle phenotypes if too few lines are analyzed, but could be circumvented by the use of isogenic lines, which appears to be the most appropriate control since gene mutation correction allows reversing disease phenotypes. ${ }^{44,45}$

We also demonstrated differentiation of our iPSC library lines not only into midbrain dopaminergic neurons but also to relatively pure astrocyte and enriched oligodendrocyte cultures. Considering that non-neuronal cells, not limited to oligodendrocytes in MSA, contribute to the PD pathology, our iPSC library will allow for

Figure 4. Tests of pluripotency applied to the induced pluripotent stem cells (iPSC) lines generated at the Cell and Stem Cell (CSC) Laboratory for CNS disease modeling, at the University of Lund, in Sweden. (a) Prior to transduction, human fibroblasts display a bipolar elongated shape. Upon transduction, putative iPSC colonies show a human pluripotent stem cell-like morphology. Scale bars represent $100 \mu \mathrm{m}$. (b) Loss and maintenance of Sendai virus in iPSC lines CSC-11A (at passage 13) and CSC-11C (at passage 13), respectively. Nuclei are counterstained with DAPI. Scale bar represents $100 \mu \mathrm{m}$. (c) Representative images of iPSC lines stained for the pluripotent markers OCT4, NANOG, TRA1-80 and SSEA4. IPSC colonies stain positive for alkaline phosphatase activity. Images are shown for iPSC line CSC-22A. Scale bars represent $100 \mu$ m. $n=2-3$ independent experiments. (d) Karyogram of iPSC line CSC-7B shows pairs of chromosomes stained using Giemsa (G-banding). (e) IPSC lines form embryoid bodies (EBs) when grown in low-adherent surfaces for 2 weeks, in WiCell Medium supplemented with FGF2 (representative image shown for iPSC line CSC-22A). Scale bar represents $100 \mu \mathrm{m} . n=2-3$ independent experiments. (f) Differentiated EBs generate cells of the three germ layers, immunopositive for alpha-fetoprotein (endoderm), smooth muscle antibody (mesoderm), and beta IIItubulin (ectoderm); nuclei are counterstained with DAPI. Scale bar represents $100 \mu \mathrm{m} . n=2-3$ independent experiments. (g) Detection of telomerase activity by the TRAP assay. (h) Upregulation of SNCA expression in clonal iPSC cell lines CSC-3A, -3B, 3G and 3S, revealed by quantitative real-time PCR. Values are normalized to house keeping gene GAPDH and calibrated to marker expression in human embryonic stem cell (hESC) line H13. Mean \pm s.e.m. shown for $n=2$ independent experiments. (i) Upregulation of SNCA expression in SNCA triplication iPSC lines revealed by quantitative real-time PCR. One-way analysis of variance (ANOVA; $P>0.0001, \mathrm{~F}_{(10 ; 49)}=30.03$ ) followed by Dunnett's multiple comparisons tests shows SNCA triplication lines have a significantly $(* * * P<0.0001)$ higher SNCA expression over non-SNCA triplication lines. Mean \pm s.e.m. (j) Higher expression of SNCA in lines generated with retrovirus transduction over those generated with Sendai virus transduction. One-way ANOVA $\left(P>0.0001, \mathrm{~F}_{(2 \cdot 29)}=58.36\right)$ followed by Tukey's multiple comparisons test shows lines reprogramed using retrovirus transduction have a significant $\left({ }^{* * *} P<0.0001\right)$ higher basal level of SNCA expression, when compared with SNCA expression levels of hESC line H13 and iPSC lines generated using Sendai virus transduction. Mean \pm s.e.m. (k) Representative images of iPSC lines differentiated towards neural, neuronal, and glial fates. IPSC lines differentiated for 12 days become midbrain neural progenitors co-expressing LMX1A and FOXA2 (shown for iPSC line CSC-1B); when progenitors are kept for 4 additional weeks in culture, they differentiate into FOXA2/tyrosine hydroxylase-expressing neurons (shown for iPSC line CSC-3G; aged 30 days in vitro). Culturing of the remaining undifferentiated neural progenitors for 5 additional weeks generates glial fibrillary acidic protein-expressing astrocytes. O4-positive oligodendrocytes can be generated from iPSC using medium devoid of retinoic acid, as we previously published. ${ }^{4}$ Nuclei are counterstained with DAPI. Scale bars represent $100 \mu \mathrm{m}$. Images are representative of $n=1-2$ independent experiments. 
disease mechanism analyses and drug screens practically with all cell types involved in development of PD. Three-dimensional cell culture models ${ }^{46-48}$ and transplantation of iPSC-derived neural cells into the rodent brain ${ }^{15,49-54}$ are still other promising strategies to be pursued using PD-iPSC library lines for revealing the contribution of genetic factors to this disease.

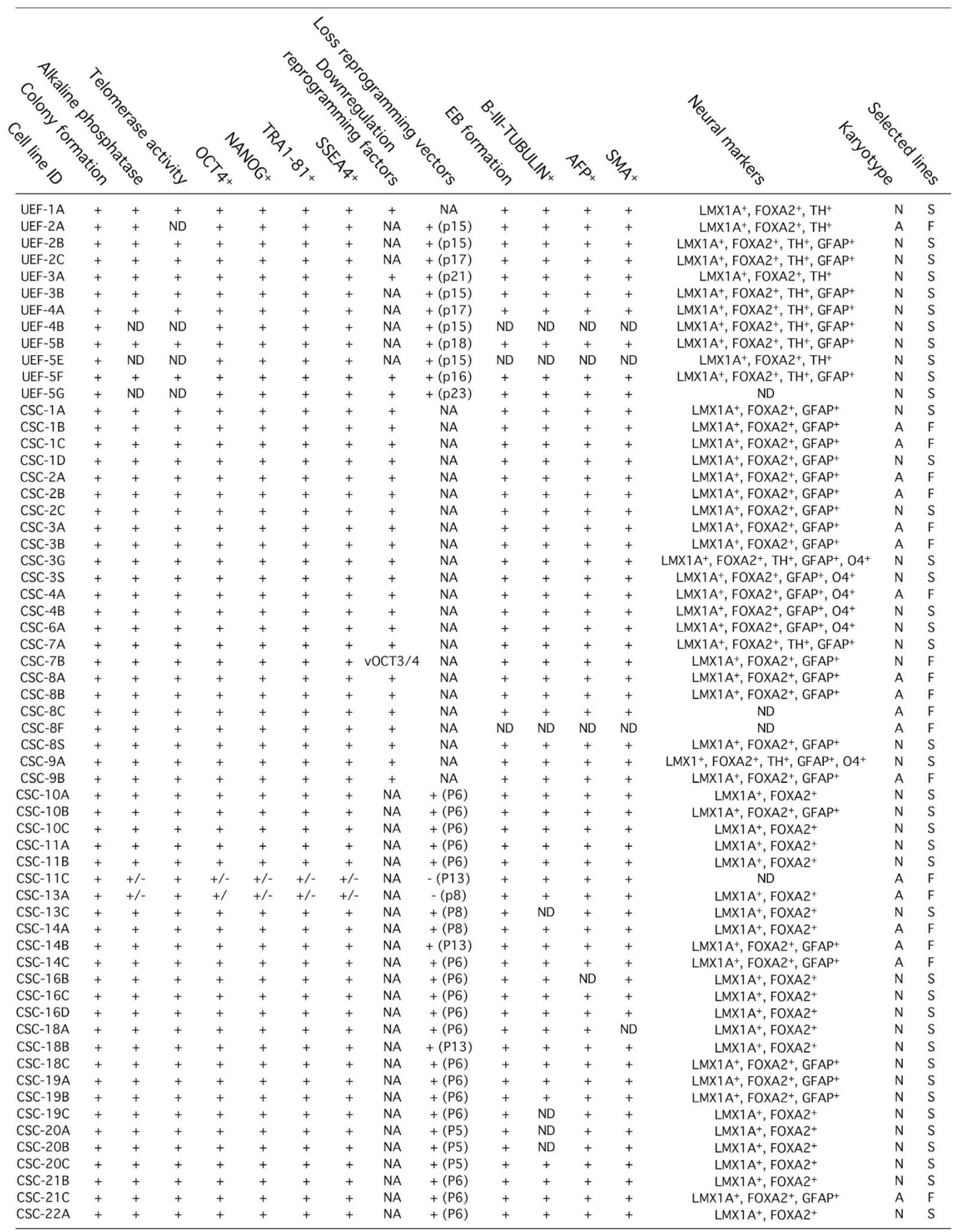

Figure 5. Summary of human induced pluripotent stem cells generated. Summary of the assays employed for characterizing the iPSC lines. The iPSC lines UEF-1 A, CSC-3A, B, G and S, -4A and B, -6A and -9A and B were previously characterized in refs. ${ }^{4,15,26-28}$ A, abnormal; $F$, failed; N, normal; NA, not applicable, ND, not determined; (Pn), passage when karyotyping was performed; S, selected; + , successfully passed; $+/-$, presence of marker of interest. 
IPSC-based models provide an unprecedented opportunity to study rare diseases of the brain where genetic causes have not been identified yet, and offer the possibility to develop new diagnostic tools for early diagnosis, as well as for the prospective stratification and recruitment of patients for future clinical trials. Importantly, iPSCs provide a source of terminally differentiated cells that can be used to develop completely humanized assays for small to high throughput or high-content screens of neuroprotective compounds ${ }^{13,17}$ and validation of drug candidates previously identified using nonrelevant cell models. ${ }^{55}$ Taking example of existing Lab-on-chip platforms, iPSC research could rapidly develop towards a Lab-on-iPSC platform or Pharmaco-iPSCellomics by person-specific iPSCs, as previously reported. ${ }^{12}$ Because of their pluripotency, iPSCs retain the ability to differentiate into multiple cell types of the three germ layers. Consequently, compounds identified as potential neuroprotective drug candidates could be tested in patient iPSC-derived hepatocytes and cardiomyocytes for possible toxicity, providing strong pre-clinical data and a higher degree of confidence prior to starting clinical trial, as well as a greater level of safety for the patients. The implementation of such ambitious projects will require the use of high-throughput platforms and stringent standard operative procedures not always available in academic institutions. It is likely that these projects will have to be conducted in large consortia, and in collaboration with industrial partners, ${ }^{56,57}$ as their cost would be enormous, especially if combinations of several drug candidates are tested.

In this study, we reprogrammed human fibroblasts from healthy subjects and patients diagnosed with PD and MSA. We applied a battery of quality control tests, which allowed us to select 41 iPSC lines for follow up studies, out of the 61 iPSC lines we initially characterized. We excluded lines that displayed abnormal karyotyping $(n=19)$, strong expression of reprogramming factor due to transgene insertion (line CSC-7B), and persistence of Sendai virus (lines CSC-11C and CSC-13A). In summary, we obtained 8 "good" control iPSC lines and 33 "good" disease iPSC lines (Figure 5).

We are continuously generating new iPSC lines using somatic cells from individuals diagnosed with synucleinopathies, including idiopathic PD cases. We have also extended our capabilities to other neurodegenerative diseases such as Alzheimer's disease. These models will allow us to identify mechanisms that are truly disease specific to those commonly engaged in an adaptive response to neurodegeneration. Such approach, together with ongoing efforts in the field, should allow gaining insights into neurodegenerative processes, and help identify proper targets for therapeutic interventions and allow "GWA"-like iPSC studies for the discovery of new biomarkers and patient stratification.

\section{MATERIALS AND METHODS}

Use of animals and human samples

All procedures were conducted in accordance with national and European Union directives. The generation of human iPSC lines using viral-mediated gene delivery was approved by the ethical committee for the use of laboratory animals at Lund University and the ethical committee on Research Ethics of Northern Savo Hospital district, Finland, as well as the Swedish Work Environment Authority (Arbetsmiljö verket).

\section{Statistical analyses}

All quantitative data was analyzed using Prism 6.0 (GraphPad, La Jolla, CA, USA). Sample groups were subjected to one-way analysis of variance and unpaired t-test. A $P$-value of $<0.05$ was considered significant.

\section{Experimental procedures}

Details methods are described in Supplementary Methods, and published in refs 4,15,26 and 37 .

\section{ACKNOWLEDGMENTS}

We gratefully thank AnnaKarin Oldén, Marianne Juhlin, Anna Hammarberg, and Dorte Lyholmer for their outstanding technical assistance, and Riitta Kauppinen, Elina Reinikainen, Laila Kaskela and Eila Korhonen for their excellent assistance in maintenance and characterization of hiPSC cultures, the staff at the cytogenetic units for preparing the samples for karyotyping and A.C. McCourt for quantitative real-time PCR assistance and analysis. We want to thank Seppo Heinonen (Kuopio University Hospital, Finland) for providing skin samples of controls and Hans R. Widmer (University of Bern, Switzerland) for providing human embryonic tissue. We are also thankful to the biobanks Coriell, and the 'Cell Line and DNA Biobank from Patients affected by Genetic Diseases' (Istituto G. Gaslini, Genova, Italy) and the 'Parkinson Institute Biobank' (Milan, Italy, http://www.parkinsonbiobank.com), members of the Telethon Network of Genetic Biobanks (project no. GTB12001) funded by Telethon Italy, for providing fibroblasts samples. This work was supported by the Strategic Research Environment MultiPark-multidisciplinary research on Parkinson's disease at Lund University, TEKES - the Finnish Funding Agency for Innovation, and through external grants to L.R. from the Bergvall foundation, the Jeanssons foundation, the Swedish Parkinson foundation (Parkinsonfonden), the Holger Crafoord foundation, the Segerfalk foundation, the Åke Wibergs foundation, the Greta och Johan Kocks foundation, and donations for science, medicine and technology at Fysiografen in Lund, and the Thelma Zoegas foundation, external grants to M.M. from the Danish Parkinson Foundation and the Lundbeck Foundation and to J.K. from the Saastamoinen Foundation, the Sigrid Juselius Foundation, The Finnish Parkinson Foundation and Academy of Finland. L.R. is a young investigator supported by MultiPark-Strategic Research Environment at Lund University, funded by the Swedish government. L.R. and M.M. are partners of BrainStem-Stem Cell Center of Excellence in Neurology, funded by Innovation Fund Denmark. J.K. and L.R. are coordinator and vice-coordinator, respectively, of a Joint Programme for Neurodegenerative Disease (JPND) research co-funded by the EU Research and Innovation Programme Horizon 2020 through the ERA-NET co-fund scheme.

\section{CONTRIBUTIONS}

S.H. carried out generation of iPSC, expansion, characterization, telomerase activity, iPSC differentiation into midbrain floor-plate progenitors, dopamine neurons and astrocytes, data collection, manuscript writing. Š.L. carried out iPSC characterization, telomerase activity, EB culture and three germ layers formation, loss of reprogramming factors, alkaline phosphatase activity, differentiation toward dopaminergic neurons and their characterization, astrocytes characterization, summarizing the data, manuscript writing. M.C. carried out iPSC expansion, characterization, EB formation, differentiation towards three germ layers, iPSC differentiation into midbrain floor-plate progenitors, data collection. K.A.P. carried out transduction and cloning of iPSCS, characterization, summarizing the data, and manuscript writing. C.A. and M.D. carried out IPSC expansion, characterization, quantitative real-time $P C R$ and oligodendrocyte differentiation. L.O. carried out fibroblast cultures, karyotyping, transduction and cloning of iPSCs, PCR, realtime PCR. M.R. and M.O. carried out fibroblast cultures, iPS cultures and cell line characterizations, astrocyte differentiation, immunocytochemistry and quantitative real-time PCR. A.C. carried out generation karyograms and supervision of analysis. M. M. and S.G. helped in provision of study material and editing of the manuscript. J.K. collected the data, wrote the manuscript, provided financial support, and gave final approval of manuscript. L.R. assisted with the generation and characterization of the iPSCs, collected the data, wrote the manuscript, provided financial support, and gave final approval of manuscript.

\section{COMPETING INTERESTS}

The authors declare no conflict of interest.

\section{REFERENCES}

1. Polymeropoulos, M. H. et al. Mutation in the alpha-synuclein gene identified in families with Parkinson's disease. Science 276, 2045-2047 (1997).

2. Ross, O. A. et al. Genomic investigation of alpha-synuclein multiplication and Parkinsonism. Ann. Neurol. 63, 743-750 (2008).

3. Wenning, G. K., Colosimo, C., Geser, F. \& Poewe, W. Multiple system atrophy. Lancet Neurol. 3, 93-103 (2004).

4. Djelloul, M. et al. Alpha-synuclein expression in the oligodendrocyte lineage: an in vitro and in vivo study using rodent and human models. Stem Cell Rep. 5, 174-184 (2015).

5. Braak, H. et al. Staging of brain pathology related to sporadic Parkinson's disease. Neurobiol. Aging 24, 197-211 (2003).

6. Chung, C. Y. et al. Identification and rescue of alpha-synuclein toxicity in Parkinson patient-derived neurons. Science 342, 983-987 (2013). 
7. Luk, K. C. et al. Intracerebral inoculation of pathological alpha-synuclein initiates a rapidly progressive neurodegenerative alpha-synucleinopathy in mice. J. Exp. Med. 209, 975-986 (2012).

8. Lee, H. J. et al. Direct transfer of alpha-synuclein from neuron to astroglia causes inflammatory responses in synucleinopathies. J. Biol. Chem. 285, 9262-9272 (2010).

9. Hallett, P. J. et al. Long-term health of dopaminergic neuron transplants in Parkinson's disease patients. Cell Rep. 7, 1755-1761 (2014).

10. Brundin, P., Melki, R. \& Kopito, R. Prion-like transmission of protein aggregates in neurodegenerative diseases. Nat. Rev. Mol. Cell Biol. 11, 301-307 (2010).

11. Takahashi, K. et al. Induction of pluripotent stem cells from adult human fibroblasts by defined factors. Cell 131, 861-872 (2007).

12. Inoue, H. \& Yamanaka, S. The use of induced pluripotent stem cells in drug development. Clin. Pharmacol. Ther. 89, 655-661 (2011).

13. Lamas, N. J. et al. Neurotrophic requirements of human motor neurons defined using amplified and purified stem cell-derived cultures. PLOS ONE 9, e110324 (2014).

14. Okano, H. \& Yamanaka, S. iPS cell technologies: significance and applications to CNS regeneration and disease. Mol. Brain 7, 22 (2014).

15. Pomeshchik, Y. et al. Transplanted human induced pluripotent stem cell-derived neural progenitor cells do not promote functional recovery of pharmacologically immunosuppressed mice with contusion spinal cord injury. Cell Transplant. 24, 1799-1812 (2015).

16. Sirenko, O., Hesley, J., Rusyn, I. \& Cromwell, E. F. High-content high-throughput assays for characterizing the viability and morphology of human iPSC-derived neuronal cultures. Assay Drug Dev. Technol. 12, 536-547 (2014).

17. Yang, Y. M. et al. A small molecule screen in stem-cell-derived motor neurons identifies a kinase inhibitor as a candidate therapeutic for ALS. Cell Stem Cell 12 , 713-726 (2013)

18. Cooper, O. et al. Pharmacological rescue of mitochondrial deficits in iPSC-derived neural cells from patients with familial Parkinson's disease. Sci. Translat. Med. 4, 141 ra190 (2012)

19. Nguyen, H. N. et al. LRRK2 mutant iPSC-derived DA neurons demonstrate increased susceptibility to oxidative stress. Cell Stem Cell 8, 267-280 (2011).

20. Ren, Y. et al. Parkin mutations reduce the complexity of neuronal processes in iPSC-derived human neurons. Stem Cells 33, 68-78 (2015).

21. Shaltouki, A. et al. Mitochondrial alterations by PARKIN in dopaminergic neurons using PARK2 patient-specific and PARK2 knockout isogenic iPSC lines. Stem Cell Rep. 4, 847-859 (2015).

22. Schondorf, D. C. et al. iPSC-derived neurons from GBA1-associated Parkinson's disease patients show autophagic defects and impaired calcium homeostasis. Nat. Commun. 5, 4028 (2014).

23. Woodard, C. M. et al. iPSC-derived dopamine neurons reveal differences between monozygotic twins discordant for Parkinson's disease. Cell Rep. 9, 1173-1182 (2014).

24. Boulting, G. L. et al. A functionally characterized test set of human induced pluripotent stem cells. Nat. Biotechnol. 29, 279-286 (2011).

25. Li, Y. et al. A comprehensive library of familial human amyotrophic lateral sclerosis induced pluripotent stem cells. PLoS ONE 10, e0118266 (2015).

26. Puttonen, K. A. et al. Improved method of producing human neural progenitor cells of high purity and in large quantities from pluripotent stem cells for transplantation studies. Cell Transplant. 22, 1753-1766 (2013).

27. Qu, C. et al. Chondrogenic differentiation of human pluripotent stem cells in chondrocyte co-culture. Int. J. Biochem. Cell Biol. 45, 1802-1812 (2013).

28. Puttonen, K. A. et al. Generation of functional neuromuscular junctions from human pluripotent stem cell lines. Front. Cell. Neurosci. 9, 473 (2015).

29. Hussein, S. M. et al. Copy number variation and selection during reprogramming to pluripotency. Nature 471, 58-62 (2011).

30. Pera, M. F. The proteomes of native and induced pluripotent stem cells. Nat. Methods 8, 807-808 (2011).

31. Kriks, S. et al. Dopamine neurons derived from human ES cells efficiently engraft in animal models of Parkinson's disease. Nature 480, 547-551 (2011).

32. $\mathrm{Xi}$, J. et al. Specification of midbrain dopamine neurons from primate pluripotent stem cells. Stem Cells 30, 1655-1663 (2012).

33. Krencik, R. \& Zhang, S. C. Directed differentiation of functional astroglial subtypes from human pluripotent stem cells. Nat. Protoc. 6, 1710-1717 (2011)

34. Beers, J. et al. A cost-effective and efficient reprogramming platform for largescale production of integration-free human induced pluripotent stem cells in chemically defined culture. Sci. Rep. 5, 11319 (2015).
35. Rao, M. S. \& Malik, N. Assessing iPSC reprogramming methods for their suitability in translational medicine. J. Cell. Biochem. 113, 3061-3068 (2012).

36. Schlaeger, T. M. et al. A comparison of non-integrating reprogramming methods. Nat. Biotechnol. 33, 58-63 (2015).

37. Holmqvist, S. et al. Generation of human pluripotent stem cell reporter lines for the isolation of and reporting on astrocytes generated from ventral midbrain and ventral spinal cord neural progenitors. Stem Cell Res. 15, 203-220 (2015).

38. Takahashi, K. \& Yamanaka, S. Induction of pluripotent stem cells from mouse embryonic and adult fibroblast cultures by defined factors. Cell 126, 663-676 (2006).

39. Taranger, C. K. et al. Induction of dedifferentiation, genomewide transcriptional programming, and epigenetic reprogramming by extracts of carcinoma and embryonic stem cells. Mol. Biol. Cell 16, 5719-5735 (2005).

40. Hakelien, A. M., Landsverk, H. B., Robl, J. M., Skalhegg, B. S. \& Collas, P. Reprogramming fibroblasts to express T-cell functions using cell extracts. Nat. Biotechnol. 20, 460-466 (2002).

41. Okita, K. et al. A more efficient method to generate integration-free human iPS cells. Nat. Methods 8, 409-412 (2011).

42. Warren, L. et al. Highly efficient reprogramming to pluripotency and directed differentiation of human cells with synthetic modified mRNA. Cell Stem Cell 7, 618-630 (2010).

43. Hou, P. et al. Pluripotent stem cells induced from mouse somatic cells by smallmolecule compounds. Science 341, 651-654 (2013).

44. Ryan, S. D. et al. Isogenic human iPSC Parkinson's model shows nitrosative stressinduced dysfunction in MEF2-PGC1alpha transcription. Cell 155, 1351-1364 (2013).

45. Sanders, L. H. et al. LRRK2 mutations cause mitochondrial DNA damage in iPSCderived neural cells from Parkinson's disease patients: reversal by gene correction. Neurobiol. Dis. 62, 381-386 (2014).

46. Choi, S. H. et al. A three-dimensional human neural cell culture model of Alzheimer's disease. Nature 515, 274-278 (2014).

47. Kim, Y. H. et al. A 3D human neural cell culture system for modeling Alzheimer's disease. Nat. Protoc. 10, 985-1006 (2015)

48. Pasca, A. M. et al. Functional cortical neurons and astrocytes from human pluripotent stem cells in 3D culture. Nat. Methods 12, 671-678 (2015).

49. Avaliani, N. et al. Optogenetics reveal delayed afferent synaptogenesis on grafted human-induced pluripotent stem cell-derived neural progenitors. Stem Cells 32, 3088-3098 (2014).

50. Doerr, J. et al. Arylsulfatase A overexpressing human ipsc-derived neural cells reduce cns sulfatide storage in a mouse model of metachromatic leukodystrophy. Mol. Ther. 23, 1519-1531 (2015).

51. Qin, J. et al. Transplantation of induced pluripotent stem cells alleviates cerebral inflammation and neural damage in hemorrhagic stroke. PLOS ONE 10, e0129881 (2015).

52. Thompson, L. H. \& Bjorklund, A. Reconstruction of brain circuitry by neural transplants generated from pluripotent stem cells. Neurobiol. Dis. 79, 28-40 (2015).

53. Tornero, D. et al. Human induced pluripotent stem cell-derived cortical neurons integrate in stroke-injured cortex and improve functional recovery. Brain 136, 3561-3577 (2013)

54. Wang, S. et al. Human iPSC-derived oligodendrocyte progenitor cells can myelinate and rescue a mouse model of congenital hypomyelination. Cell Stem Cell 12, 252-264 (2013).

55. Mertens, J. et al. APP processing in human pluripotent stem cell-derived neurons is resistant to NSAID-based gamma-secretase modulation. Stem Cell Rep. 1, 491-498 (2013).

56. Brennand, K. J. et al. Creating patient-specific neural cells for the in vitro study of brain disorders. Stem Cell Rep. 5, 933-945 (2015).

57. Paull, D. et al. Automated, high-throughput derivation, characterization and differentiation of induced pluripotent stem cells. Nat. Methods 12, 885-892 (2015).

This work is licensed under a Creative Commons Attribution 4.0 International License. The images or other third party material in this article are included in the article's Creative Commons license, unless indicated otherwise in the credit line; if the material is not included under the Creative Commons license, users will need to obtain permission from the license holder to reproduce the material. To view a copy of this license, visit http://creativecommons.org/licenses/ by/4.0/

Supplementary Information accompanies the paper on the npj Parkinson's Disease website (http://www.nature.com/npjparkd) 